\title{
Plasmodium falciparum induces Foxp3hi CD4T cells independent of surface PfEMP1 expression via small soluble parasite components
}

\author{
Anja Scholzen ${ }^{1,2+}$, Brian M. Cooke ${ }^{3}$ and Magdalena Plebanski ${ }^{1}$ * \\ ' Department of Immunology, Monash University, Melbourne, VIC, Australia \\ ${ }^{2}$ Department of Medical Microbiology, Radboud university medical center, Nijmegen, Netherlands \\ ${ }^{3}$ Department of Microbiology, Monash University, Clayton, VIC, Australia
}

\section{Edited by:}

Shuo Li, Macfarlane Burnet Institute for Medical Research and Public Health, Australia

\section{Reviewed by:}

Hajime Hisaeda, Gunma University, Japan

Diana Silvia Hansen, The Walter and Eliza Hall Institute of Medical

Research, Australia

\section{${ }^{*}$ Correspondence:}

Magdalena Plebanski, Department of Immunology, Monash University, Commercial Road, Melbourne, VIC 3004, Australia

e-mail:magdalena.plebanski@ monash.edu

${ }^{\dagger}$ Present address:

Anja Scholzen, Department of Medical Microbiology, Radboud university medical center, Nijmegen, Netherlands
Elevated levels of regulatory T cells following Plasmodium infection are a well-reported phenomenon that can influence both protective and pathological anti-parasite responses, and might additionally impact on vaccine responses in acutely malaria infected individuals. The mechanisms underlying their induction or expansion by the parasite, however, are incompletely understood. In a previous study, Plasmodium falciparum infected red blood cells (iRBCs) were shown to induce effector-cytokine producing Foxp3int CD4+ T cells, as well as regulatory Foxp3hi CD4+ T cells in vitro. The aim of the present study was to determine the contribution of parasite components to the induction of Foxp3 expression in human CD4+ $T$ cells. Using the surface PfEMP1-deficient parasite line 1G8, we demonstrate that induction of Foxp3hi and Foxp3int CD4+T cells is independent of PfEMP1 expression on iRBCs. We further demonstrate that integrity of iRBCs is no requirement for the induction of Foxp3 expression. Finally, transwell experiments showed that induction of Foxp3 expression, and specifically the generation of Foxp3hi as opposed to Foxp3int CD4T cells, can be mediated by soluble parasite components smaller than $20 \mathrm{~nm}$ and thus likely distinct from the malaria pigment hemozoin. These results suggest that the induction of Foxp3hi T cells by P. falciparum is largely independent of two key immune modulatory parasite components, and warrant future studies into the nature of the Foxp3hi inducing parasite components to potentially allow their exclusion from vaccine formulations.

Keywords: Malaria, Plasmodium falciparum, regulatory T cell, Foxp3, PfEMP-1, hemozoin

\section{INTRODUCTION}

Malaria caused by infection with protozoan Plasmodium parasites is a life threatening disease that is at least partially immune mediated. Disease-contributing factors include excessive inflammatory responses and overwhelming parasite replication insufficiently controlled by anti-parasite immune responses. Regulatory $\mathrm{T}$ cells (Tregs) can suppress both protective as well as pathological adaptive immune responses, and are elevated in both human falciparum and vivax malaria as well as in murine malaria models (Scholzen et al., 2010). Although the consequences of elevated Treg levels during malaria are yet to be determined, several studies indicate that depending on the stage of infection, increased Treg levels can be protective or detrimental to the host (Finney et al., 2010; Hansen and Schofield, 2010; Scholzen et al., 2010). In addition to potentially limiting responses to parasite antigens (Ho etal., 1986; Bejon etal., 2007), elevated Treg levels during acute blood-stage malaria infection might also contribute to the reduced acquisition of immune responses to heterologous antigens, such as standard childhood vaccines (Greenwood etal., 1972; Williamson and Greenwood, 1978; Whittle etal., 1984). The mechanisms underlying the elevated levels of this important cell type during malaria, however, are incompletely understood.
We have previously dissected the host immune mechanisms contributing to the induction of effector-cytokine producing CD25+Foxp3int CD4+ $\mathrm{T}$ cells, as well as regulatory CD25+Foxp3hi CD4+ T cells that inhibited Foxp3int effector cytokine production, following $P$. falciparum-infected red blood cells (iRBCs) exposure in vitro (Scholzen et al., 2009). The parasite factors responsible for Treg induction during malaria are yet unknown. This is especially relevant as the identification of parasite-specific components relevant for Treg induction may allow the development of intervention strategies directly targeted at the parasite, and the specific exclusion or inclusion of parasite components in therapeutic or vaccine formulations (Casares and Richie, 2009; Higgins et al., 2011).

Two key parasite components that have attracted attention as modulators of immune response in both human and murine malaria are the virulence factor and variant surface antigen $P$. falciparum erythrocyte membrane protein (PfEMP)-1, and the heme degradation product and malaria pigment hemozoin. Whilst findings for PfEMP-1 are controversial, both have been shown in a number of studies to interfere with the activation and maturation of antigen-presenting cells such as monocytes and dendritic cells (Millington et al., 2006; Wykes and Good, 2008; Stevenson et al., 2011). This is particularly relevant as both in 
human in vitro studies (Scholzen et al., 2009; Finney et al., 2012; Clemente et al., 2013) and in murine models (Hisaeda et al., 2008), antigen-presenting cells are crucial mediators of Treg induction and activation by malaria parasites. The aim of the present study was therefore to determine the contribution of parasite components to the induction of Foxp3 expression in human CD4+ T cells.

\section{MATERIALS AND METHODS}

\section{P. falciparum CULTURE AND TROPHOZOITE ISOLATION}

Mycoplasma-free blood-stage parasites of P. falciparum (strain 3D7) were maintained in O+ erythrocytes in RPMI-1640 medium (JRH, Lenexa, KS, USA) supplemented with $1 \mathrm{mM}$ glutamine, $11 \mathrm{mM}$ glucose, $25 \mathrm{mM}$ HEPES, $0.2 \%(\mathrm{w} / \mathrm{v})$ sodium bicarbonate, $200 \mathrm{mM}$ hypoxanthine, $40 \mathrm{mg} / \mathrm{ml}$ gentamycin (all Sigma-Aldrich, St. Louis, MO, USA), and 0.5\% (w/v) AlbuMAX II (GIBCO, Invitrogen, Carlsbad, CA, USA) at $37^{\circ} \mathrm{C}$ in an atmosphere of $5 \% \mathrm{CO} 2$ and $1 \% \mathrm{O} 2$ in N2. Knob-expressing parasites were enriched weekly using gelofusine solution (Braun Melsungen, Germany). The 3D7derived SBP-1 knock-out parasite line [clone 1G8 (Cooke et al., 2006)] was grown under drug-pressure (2.5 nM WR99210 and $4 \mu \mathrm{M}$ Ganciclovir). Trophozoite stage parasites were isolated by density gradient centrifugation following layering onto a gradient of 40/60/80\% isotonic Percoll (Amersham Biosciences, Uppsala, Sweden). The percentage of infected erythrocytes was typically 90-100\%.

\section{PBMC ISOLATION AND IRBC:PBMC CO-CULTURE}

To examine the induction of Foxp3 expression by iRBCs, we employed the in vitro co-culture system previously validated in our laboratory (Scholzen et al., 2009). Peripheral blood mononuclear cells (PBMCs) were recovered by Ficoll-Hypaque (Amersham Biosciences) density gradient centrifugation from buffy coats [Australian Red Cross Blood Service (ARCBS), Melbourne, VIC, Australia]. The ARCBS received informed consent from all donors to use their donation for research purposes and the Monash University Human Research Ethics Committee approved the research purpose for which buffy coats were used. Autologous human serum (HS) was obtained by coagulating platelet-rich plasma from buffy coats with $0.3 \%(\mathrm{w} / \mathrm{v}) \mathrm{CaCl} 2$, followed by heat inactivation at $56 \mathrm{uC}$ for $30 \mathrm{~min}$. PBMCs were cultured in AIM-V medium (GIBCO, Invitrogen) supplemented with 5\% autologous HS alone (untreated controls), with non-infected control erythrocytes or trophozoite-stage iRBCs. An iRBC:PBMC ratio of 2:1 was chosen (Scholzen et al., 2009), calculated to reflect a clinically relevant parasitemia found in natural infections $(0.1 \%$ parasitemia or 5000 iRBC/ $\mu$ l blood) (Minigo et al., 2009; Walther et al., 2009). To obtain iRBC lysate, the integrity of iRBCs was disrupted by five rounds of freeze-thawing. In some experiments, tissue culture inserts (Anopore, NUNC, Naperville, IL, USA) were used to separate iRBCs (inside) from PBMCs (outside the transwell). A pore size of $20 \mathrm{~nm}$ was chosen to prohibit transfer of hemozoin crystals, which are on all sides larger than this cut-off (Noland et al., 2003).

\section{CELL PHENOTYPING BY FLOW CYTOMETRY}

Cells were washed with PBS and incubated with antibodies diluted in $\mathrm{PBS} / 10 \% \mathrm{HS} / 0.01 \% \mathrm{NaN} 3$ (sodium azide) for $30 \mathrm{~min}$ on ice. Surface antibodies were anti-CD4 PerCp (clone SK3), CD3 FITC (clone UCHT1), and CD25 PE (clone M-A251, all BD Biosciences). Intracellular staining with anti-Foxp3 APC (clone $\mathrm{PCH} 101$, eBiosciences) was performed using the eBioscience fixation/permeabilization buffer kit. A minimum of $10^{5}$ events in the lymphocyte gate was acquired using a FACScalibur flow cytometer for 4-color analysis and analyzed using WEASEL software (WEHI, Melbourne, VIC, Australia). Cells were gated first based on forward and side scatter to excluded dead cells and cell debris. $\mathrm{T}$ cells in the lymphocyte gate were identified based on CD3 expression, further sub-gated on CD4+ T cells (Figure 1A) and CD25+ cells then subdivided into Foxp3hi and Foxp3int cells (Figure 1B).

\section{DATA PRESENTATION AND STATISTICAL ANALYSIS}

We employed normalization onto control conditions for each donor, to be able to analyze changes in Foxp3hi or Foxp3int proportions (measured as percentage of CD4 T cells). Normalized values are referred to as fold change compared to control conditions (value 1).

Statistical analysis was carried out using GraphPad Prism software v4 (San Diego, CA, USA). Due to the small power of non-parametric tests to detect differences in small sample sizes, all tests were chosen to be parametric. $P$ values between two groups were determined by two-tailed paired Student's $t$-test. Three or more groups were compared by repeated-measures oneway ANOVA, followed by Tukey's multiple comparison post test. A $p<0.05$ was considered significant.

\section{RESULTS}

We firstly addressed the question, whether the induction of Foxp3 expression in CD4+ $\mathrm{T}$ cells was dependent on interactions between iRBC surface-expressed PfEMP-1 and corresponding receptors on peripheral blood mononuclear cells such as monocytes using a recently established 3D7-derived surface PfEMP1 deficient parasite line. This parasite line lacks, due to targeted gene disruption, expression of skeleton-binding protein 1 (SBP-1), a Maurer's cleft-associated protein essential for the transport of PfEMP-1 to the iRBC surface (Cooke et al., 2006). When co-cultured with PBMC for 6 days, surface PfEMP-1 deficient $1 \mathrm{G} 8$ parasites induced proportions of CD25+Foxp3hi and CD25+Foxp3int cells that were not significantly different from those induced by wild type 3D7 iRBCs (Figure 1, one-way ANOVA with Tukey's post-test). This indicates that induction of Foxp3 expression in CD4+ $\mathrm{T}$ cells is independent of surface PfEMP-1 expression on iRBCs and therefore surface PfEMP-1-host immune receptor interaction.

Since two previous studies have used iRBC lysate or soluble extract for Foxp3 induction in vitro (Finney et al., 2012; Clemente et al., 2013), we first assessed there was a difference between intact and lysed iRBC in their ability to induce CD25+Foxp3 expressing CD4+ T cells. When comparing Foxp3 induction in PBMC co-cultures with either intact iRBCs or iRBC lysate, iRBC lysate was nearly as effective in inducing both CD25+Foxp3hi and CD25+Foxp3int CD4+ T cells (Figure 2), with no significant difference between the two for neither absolute percentages or 
A

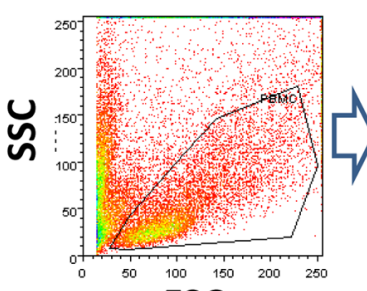

FSC

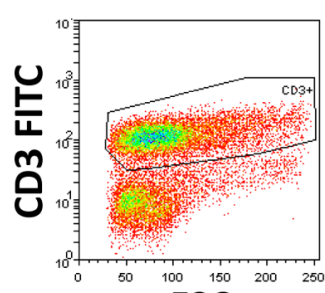

FSC

B
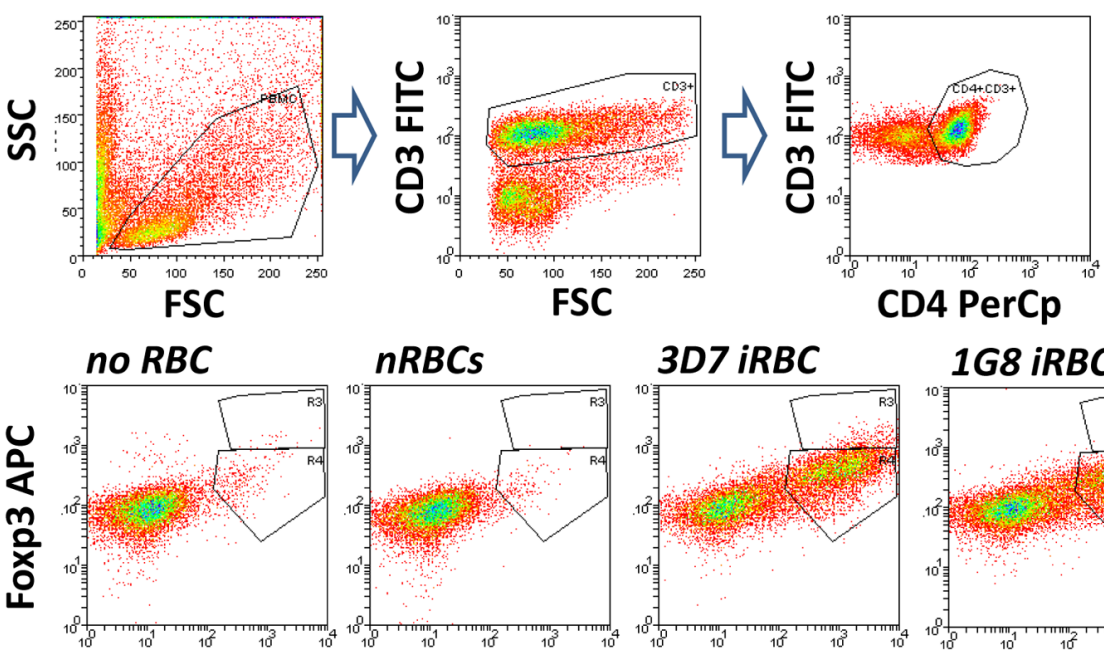

CD4 PerCp

CD25 PE

C

CD25+Foxp3int

\section{CD25+Foxp3hi}

$1 G 8$ iRBC
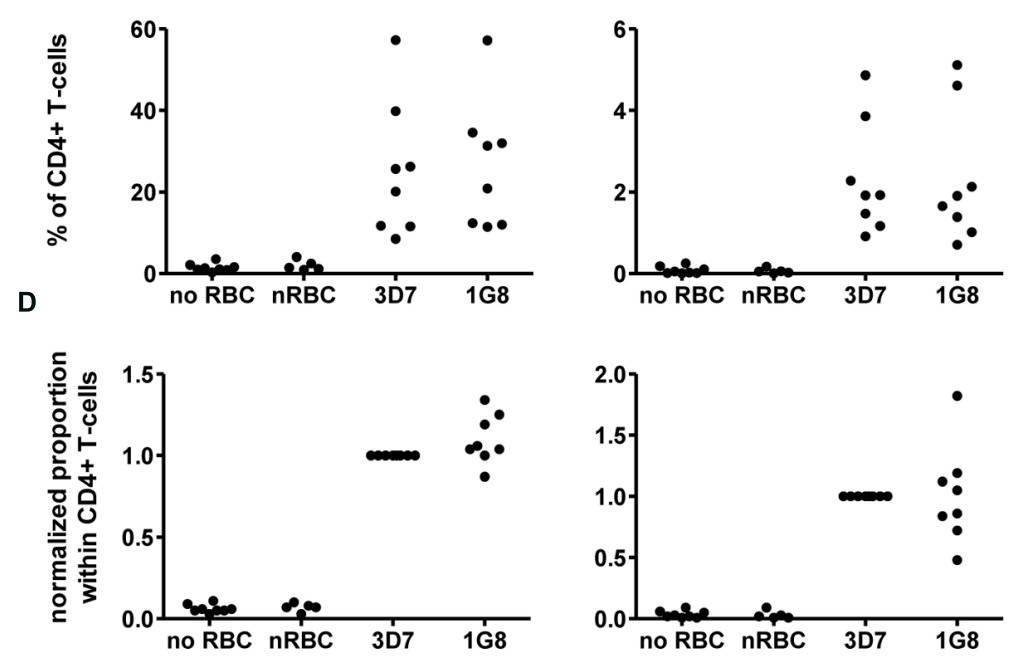

FIGURE 1 | Infected red blood cell (iRBC)-mediated Foxp3 expression is independent of surface PfEMP-1 expression. PBMC were cultured alone or in the presence of normal (nRBC) or infected RBCs (iRBC) at an RBC:PBMC ratio of 2:1. RBCs were infected with either the parental strain 3D7 or the SBP-1 knock-out parasite line 1G8. On day 6, cells were harvested and analyzed by flow cytometry. (A) Lymphocytes were selected by FSC/SSC gating and further gated based on CD3 and CD4 staining. CD4+ T cells were then analyzed based on CD25 and Foxp3 expression. (B) Representative dots plots for PBMC from one donor stimulated with 3D7 and $1 \mathrm{G} 8$ parasites. (C) Proportions of Foxp3hi and Foxp3int CD4+CD25+T cells determined as a percentage of $\mathrm{CD} 3+\mathrm{CD} 4+\mathrm{T}$ cells for eight donors in six independent experiments. (D) Values of all conditions were normalized for each individual donor on proportions induced by 3D7 iRBCs. Vertical bars represent median values. after normalization. Therefore, iRBC integrity is indeed not a prerequisite for the Foxp3 induction in $\mathrm{CD} 4+\mathrm{T}$ cells upon parasite exposure.

To further investigate the possibility that large intracellular components such as hemozoin crystals interacting with monocytes were contributing to Foxp3 induction, we employed tissue culture inserts to separate iRBCs (inside) and PBMCs (outside the transwell). We specifically chose a pore size of $20 \mathrm{~nm}$ to prohibit transfer of large parasite components, including membrane fragments and intact hemozoin crystals, which are larger than $20 \mathrm{~nm}$ in diameter on either side of their brick-like cuboidal body (Noland et al., 2003), between the two chambers. Accordingly, in this transwell setting we found no light-microscopic evidence of hemozoin incorporation into monocytes (which in direct co-cultures are typically filled with dark hemozoin material, data not shown). As shown in Figure 3, similar to direct co-cultures, iRBC separated from PBMC by a transwell were also capable of inducing Foxp3 expression in CD4+ $\mathrm{T}$ cells. Importantly, in four out of five donors Foxp3hi CD4+ T cells were still induced at levels comparable to direct co-culture (Figure 3), while induction of Foxp3int CD4+ T cells was reduced in all five donors tested (Figures 3B,C; 20-31\% compared to direct co-culture; $p<0.001$, one-way ANOVA with Tukey's post-test). As a result, there was a trend that exposure of PBMC to soluble iRBC components 
A

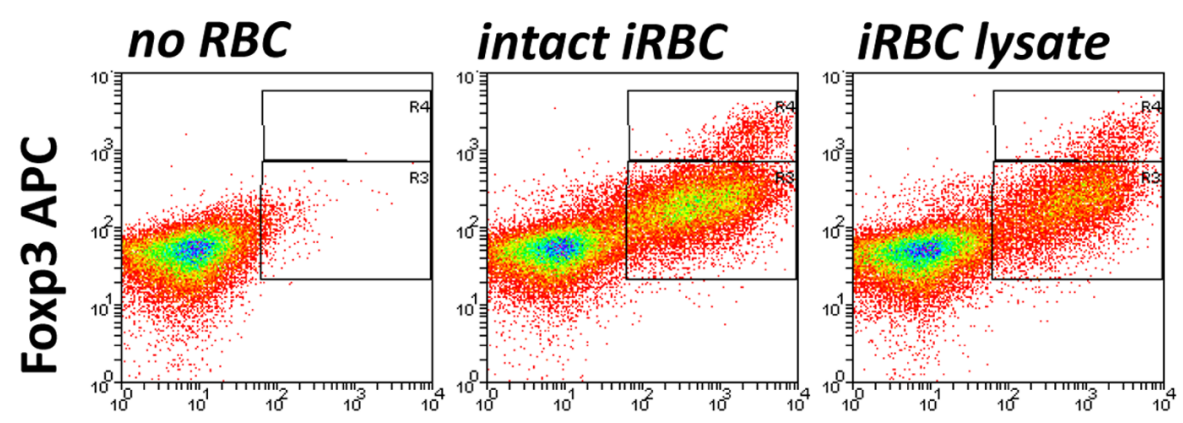

CD25 PE

B

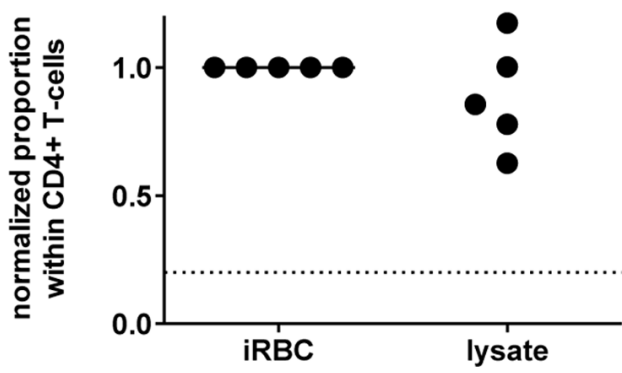

CD25+Foxp3hi
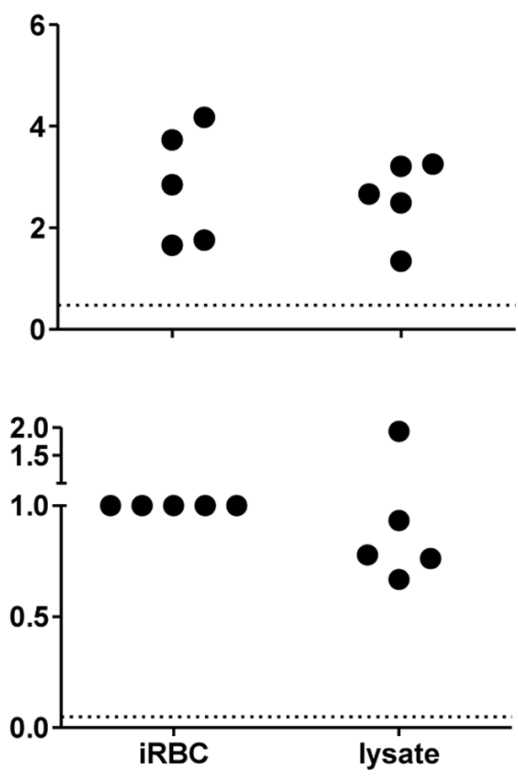

FIGURE 2 | iRBC-mediated Foxp3 expression does not require intact iRBCs. PBMC were cultured alone or in the presence of normal (nRBC) or infected $R B C s$ (iRBC) at an RBC:PBMC ratio of 2:1. PBMC were co-cultured with equal amounts of either intact 3D7 iRBCs, or 3D7 iRBC lysate resulting from five rounds of freezing and thawing. (A) Representative dots plots for PBMCs from one donor stimulated with intact iRBCs versus iRBC lysate.
(B) Proportions of Foxp3hi and Foxp3int CD4+CD25+T cells were determined on day 6 as a percentage of $C D 3+C D 4+T$ cells for five donors in two independent experiments. (C) Values of all conditions were normalized for each individual donor on proportions induced by intact iRBCs. Vertical bars represent median values. Dotted lines show the upper 95\% confidence interval of the mean of uRBC-stimulated control cultures. smaller than $20 \mathrm{~nm}$ instead of complete iRBC enhanced the ratio of Foxp3hi:Foxp3int cells within the CD4+CD25+ population (Figure 3D).

\section{DISCUSSION}

In this study, we show that $P$. falciparum iRBCs can induce Foxp3hi CD4 T cells independent of surface-expressed PfEMP-1 via soluble parasite components smaller than $20 \mathrm{~nm}$.

Similar to our finding that induction of Foxp3 expression is independent of iRBC surface PfEMP-1 expression and contact with intact iRBCs, a recent study demonstrated that human monocyte-derived DC maturation can be inhibited by $P$. falciparum independent of surface PfEMP1 expression and also across a transwell (Elliott et al., 2007). Using a murine malaria model, Orengo etal. (2008) reported that inhibition of murine
DC maturation following $P$. yoelii infection was also mediated by a yet unidentified soluble factor. Future studies are now needed to further identify the soluble factor mediating these immunomodulatory effects of the parasite. Importantly, monocytes or monocytes-derived DCs were previously shown to be required to drive the induction of Foxp3 expression in human CD4 T cells upon $P$. falciparum exposure in vitro (Scholzen et al., 2009; Clemente et al., 2013) and DCs activated Tregs in P. yoelii infected mice (Hisaeda etal., 2008). Future studies may therefore address the question whether it may even be the same mechanism by which the parasite modulates not only with monocyte and DC function, but also initiates the induction of Tregs.

Previous studies have used either intact iRBCs (Scholzen et al., 2009), iRBC lysate (Finney et al., 2012), or the soluble fraction 
A

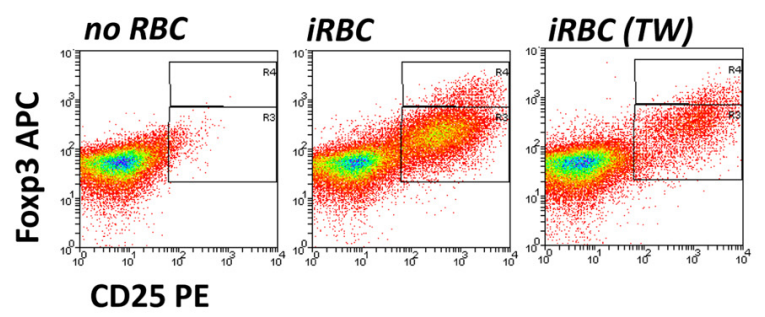

B
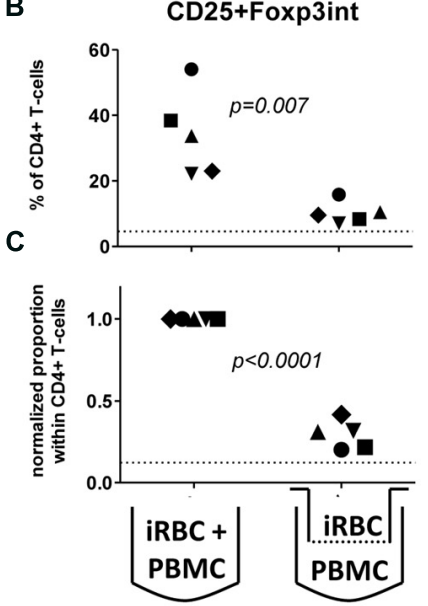

D

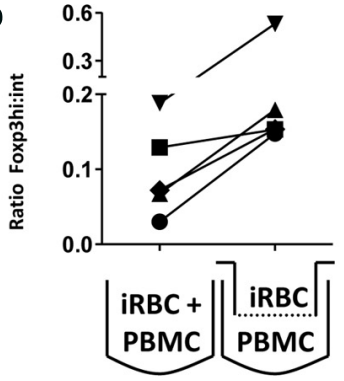

FIGURE 3 | Foxp3hi and to a lesser degree Foxp3int induction is mediated by soluble iRBC components smaller than $20 \mathbf{~ n m}$. To exclude large membrane fragments and intact hemozoin crystals and to examine the contribution of small soluble molecules derived from iRBCs to Foxp3 induction, intact $\mathrm{RBC}$ were separated from PBMC through a transwell membrane (pore size $20 \mathrm{~nm}$ ). (A) Representative dots plots for PBMCs from one donor cultured in direct contact with iRBCs compared to transwell-separated iRBCs. (B) Proportions of Foxp3hi and Foxp3int $C D 4+C D 25+T$ cells were determined on day 6 as a percentage of $C D 4+T$ cells for five donors in two independent experiments. (C) Values of all conditions were normalized for proportions induced by direct co-culture with iRBCs for each donor. Vertical bars represent median values. (D) The ratio of Foxp3hi:Foxp3int cells within the CD4+CD25+T cell population was calculated for each individual donor in direct or transwell separated co-cultures. Vertical bars represent median values. Individual donors are identified by unique symbols. Dotted lines show the upper $95 \%$ confidence interval of the mean of URBC-stimulated control cultures.

of iRBC lysate (Clemente et al., 2011) as a stimulus for Foxp3 expression in human $\mathrm{CD} 4 \mathrm{~T}$ cells in vitro. In this direct sideby-side comparison we show that Foxp3 induction by iRBC lysate is indeed comparable to intact $\mathrm{iRBC}$. Therefore, if direct cell-cell interaction between immune cells and iRBCs are not a pre-requisite for Treg induction, which is instead mediated by soluble factors resulting from schizont rupture, then Treg induction can also occur at distant sites clear of measurable parasitemia.

For instance, while mature trophozoite-stage $P$. falciparum parasites are typically sequestered in the microvasculature (to avoid clearance in the spleen), soluble parasite components released upon parasite rupture would have access to monocytes and $\mathrm{T}$ cells in the spleen. Moreover, soluble parasite components might also mediate Treg induction in more distant sites outside the circulation, such as in lymph nodes. In as how far this occurs, and whether this would affect vaccination-induced immune responses during acute blood-stage malaria infection (Greenwood et al., 1972; Williamson and Greenwood, 1978; Whittle et al., 1984), remains to be determined. Finally, such small parasite components may be able to cross the placental barrier and thus explain and contribute to the induction of Tregs in cord blood even in the absence of direct cord blood parasitemia. Indeed, in some studies levels of Tregs have been found to be elevated in neonates born to mothers who had experienced malaria episodes during pregnancy (Brustoski et al., 2006; Mackroth et al., 2011), while in other studies, ex vivo cord blood Treg frequencies were unaffected by placental malaria and only increased only upon in vitro stimulation with iRBC extract (Flanagan et al., 2010; Soulard et al., 2011). It is yet unclear whether Treg induction during a single Plasmodium infection predisposes the immune system to heightened regulatory responses at the next encounter with the parasite, but in utero exposure to Treg-inducing parasite components may prime the fetus's immune system to respond with a less inflammatory response upon re-exposure to malaria-antigens (Malhotra et al., 2009; Flanagan et al., 2010). Moreover, such malaria-induced immune modulation might also be one explanation for observations of reduced vaccination-responses in children born to women with placental-malaria (Labeaud et al., 2009; Walther et al., 2012).

Hemozoin has been shown in several studies to activate TLR9 (Shio et al., 2010). Moreover, DCs activated via TLR9 [either by iRBCs in mice (Hisaeda et al., 2008) or CpG DNA in human (Moseman etal., 2004)] can induce/activate Tregs. These findings indicate a potential role for hemozoin in Treg induction. It remains to be formally shown whether iRBC-purified or synthetic hemozoin contributes to the induction of Tregs by $P$. falciparum iRBCs. Our results from experiments using transwell inserts with a $20 \mathrm{~nm}$ pore size to separate PBMCs and iRBCs, however, suggest that hemozoin crystals do not have a major contribution to the generation of Foxp3hi T cells: Intact hemozoin crystals are larger than $20 \mathrm{~nm}$ (Noland et al., 2003) and therefore unlikely to have crossed the $20 \mathrm{~nm}$ sized pores of the transwell inserts used in this study. We adoped a transwell approach to prohibit the transfer of intact hemozoin crystals after schizont rupture, since it is currently technically not possible to deplete iRBC extracts of hemozoin without denaturing other lysate components and antigens (Coban et al., 2010). And while we cannot rule out that smaller hemozoin crystal fragments might have crossed the transwell border, the TLR9 binding capacity of hemozoin has recently been shown to be restricted to a crystal size range of 50-200 nm, while hemin molecules smaller than $50 \mathrm{~nm}$ were ineffective (Coban et al., 2010). 
A curious finding was the trend towards a favored induction of Foxp3hi over Foxp3int $\mathrm{T}$ cells when components larger than $20 \mathrm{~nm}$ were excluded from the co-culture using transwell membranes. Whilst these data require further analysis in future studies, it is tempting to speculate that small soluble parasite components are preferentially driving Foxp3hi induction, whilst Foxp3int effector $\mathrm{T}$ cells may rely on a greater pool of membrane associated antigens. We have previously shown that although iRBC-mediated induction of Foxp3hi T cells does rely on T effector-produced IL-2 and is further driven by cytokines such as IL-10 and TGF $\beta$, in itself it is not dependent on MHC class II antigen-presentation and those cells therefore not necessarily malaria antigen-specific (Scholzen et al., 2009). The current data are in line with this finding, suggesting that activation of $\mathrm{T}$ cells in an environment of direct contact of antigen-presenting cells with intact iRBCs might result in a more efficient induction of (Foxp3int) effector $\mathrm{T}$ cells in a parasite antigen-specific manner. In contrast, induction of Foxp3hi $\mathrm{T}$ cells with a $\mathrm{T}$ regulatory phenotype may also occur independent of membrane-associated antigens at a distant site, mediated by cytokines acting in concert with circulating soluble parasite molecules. Further research is now necessary to determine the nature and mechanism of action of these soluble parasite components.

To conclude, our results indicate that the induction of Foxp3hi regulatory $\mathrm{T}$ cells by $P$. falciparum may be largely independent of two key immunomodulatory parasite components, namely the surface protein PfEMP1 and the malaria pigment hemozoin and warrant future studies into the nature of the Foxp3hi inducing parasite components. Furthermore, similar to their distinct cytokine requirements (Scholzen et al., 2009), Foxp3int effectorlike and Foxp3hi regulatory-like CD4+ T cells appear to rely on different parasite components for their induction. These findings merit future in-depth studies to identify the parasite components responsible for regulatory versus effector $\mathrm{T}$ cell induction. Identification of such parasite components will be important to ensure appropriate exclusion or inclusion of such parasite components from vaccine formulations.

\section{AUTHOR CONTRIBUTIONS}

Conceived and designed the experiments: Anja Scholzen, Magdalena Plebanski. Performed the experiments and analyzed the data: Anja Scholzen. Contributed essential reagents/materials/analysis tools: Brian M. Cooke, Magdalena Plebanski. Interpreted data and wrote the paper: Anja Scholzen, Magdalena Plebanski.

\section{ACKNOWLEDGMENTS}

This work was supported by the Australian NHMRC (Brian M. Cooke and Magdalena Plebanski), the Howard Hughes Medical Institute International Scholar's Program (support to Magdalena Plebanski). AS was a recipient of an Endeavour International Postgraduate Research Scholarship and a Melbourne International Research Scholarship at the University of Melbourne, Department of Medicine. The funders had no role in study design, data collection and analysis, decision to publish, or preparation of the manuscript. Red blood cells for P. falciparum culture and buffy coats were kindly provided by the Australian Red Blood Cross Service, Melbourne, Australia.

\section{REFERENCES}

Bejon, P., Mwacharo, J., Kai, O., Todryk, S., Keating, S., Lowe, B., et al. (2007). The induction and persistence of T cell IFN-gamma responses after vaccination or natural exposure is suppressed by Plasmodium falciparum. J. Immunol. 179, 4193-4201.

Brustoski, K., Moller, U., Kramer, M., Hartgers, F. C., Kremsner, P. G., Krzych, U., et al. (2006). Reduced cord blood immune effector-cell responsiveness mediated by CD4+ cells induced in utero as a consequence of placental Plasmodium falciparum infection. J. Infect. Dis. 193, 146-154. doi: 10.1086/ 498578

Casares, S., and Richie, T. L. (2009). Immune evasion by malaria parasites: a challenge for vaccine develop. Curr. Opin. Immunol. 21, 321-330. doi: 10.1016/j.coi.2009.05.015

Clemente, A., Caporale, R., Sannella, A. R., Majori, G., Severini, C., Fadigati, G., et al. (2011). Plasmodium falciparum soluble extracts potentiate the suppressive function of polyclonal $\mathrm{T}$ regulatory cells through activation of TGFbetamediated signals. Cell. Microbiol. 13, 1328-1338. doi: 10.1111/j.1462-5822.2011. 01622.x

Clemente, A. M., Fadigati, G., Caporale, R., Marchese, D. G., Castronovo, G., Sannella, A. R., et al. (2013). Modulation of the immune and inflammatory responses by Plasmodium falciparum schizont extracts: role of myeloid dendritic cells in effector and regulatory functions of CD4+ lymphocytes. Infect. Immun. 81, 1842-1851. doi: 10.1128/IAI.01226-12

Coban, C., Igari, Y., Yagi, M., Reimer, T., Koyama, S., Aoshi, T., et al. (2010). Immunogenicity of whole-parasite vaccines against Plasmodium falciparum involves malarial hemozoin and host TLR9. Cell Host Microbe 7, 50-61. doi: 10.1016/j.chom.2009.12.003

Cooke, B. M., Buckingham, D. W., Glenister, F. K., Fernandez, K. M., Bannister, L. H., Marti, M., et al. (2006). A Maurer's cleft-associated protein is essential for expression of the major malaria virulence antigen on the surface of infected red blood cells. J. Cell Biol. 172, 899-908. doi: 10.1083/jcb.2005 09122

Elliott, S. R., Spurck, T. P., Dodin, J. M., Maier, A. G., Voss, T. S., Yosaatmadja, F., et al. (2007). Inhibition of dendritic cell maturation by malaria is dose dependent and does not require Plasmodium falciparum erythrocyte membrane protein 1. Infect. Immun. 75, 3621-3632. doi: 10.1128/IAI.00095-07

Finney, O. C., Lawrence, E., Gray, A. P., Njie, M., Riley, E. M., and Walther, M. (2012). Freeze-thaw lysates of Plasmodium falciparum-infected red blood cells induce differentiation of functionally competent regulatory $\mathrm{T}$ cells from memory T cells. Eur. J. Immunol. 42, 1767-1777. doi: 10.1002/eji.2011 42164

Finney, O. C., Riley, E. M., and Walther, M. (2010). Regulatory T cells in malaria - friend or foe? Trends Immunol. 31, 63-70. doi: 10.1016/j.it.2009. 12.002

Flanagan, K. L., Halliday, A., Burl, S., Landgraf, K., Jagne, Y. J., Noho-Konteh, F., et al. (2010). The effect of placental malaria infection on cord blood and maternal immunoregulatory responses at birth. Eur. J. Immunol. 40, 1062-1072. doi: 10.1002/eji.200939638

Greenwood, B. M., Bradley-Moore, A. M., Bryceson, A. D., and Palit, A. (1972). Immunosuppression in children with malaria. Lancet 1, 169-172. doi: 10.1016/S0140-6736(72)90569-7

Hansen, D. S., and Schofield, L. (2010). Natural regulatory T cells in malaria: host or parasite allies? PLoS Pathog. 6:e1000771. doi: 10.1371/journal.ppat.10 00771

Higgins, S. J., Kain, K. C., and Liles, W. C. (2011). Immunopathogenesis of falciparum malaria: implications for adjunctive therapy in the management of severe and cerebral malaria. Expert Rev. Anti. Infect. Ther. 9, 803-819. doi: 10.1586/eri.11.96

Hisaeda, H., Tetsutani, K., Imai, T., Moriya, C., Tu, L., Hamano, S., et al. (2008). Malaria parasites require TLR9 signaling for immune evasion by activating regulatory T cells. J. Immunol. 180, 2496-2503.

Ho, M., Webster, H. K., Looareesuwan, S., Supanaranond, W., Phillips, R. E., Chanthavanich, P., etal. (1986). Antigen-specific immunosuppression in human malaria due to Plasmodium falciparum. J. Infect. Dis. 153, 763-771. doi: 10.1093/infdis/153.4.763 
Labeaud, A. D., Malhotra, I., King, M. J., King, C. L., and King, C. H. (2009). Do antenatal parasite infections devalue childhood vaccination? PLoS Negl. Trop. Dis. 3:e442. doi: 10.1371/journal.pntd.0000442

Mackroth, M. S., Malhotra, I., Mungai, P., Koech, D., Muchiri, E., and King, C. L. (2011). Human cord blood CD4+CD25hi regulatory T cells suppress prenatally acquired T cell responses to Plasmodium falciparum antigens. J. Immunol. 186, 2780-2791. doi: 10.4049/jimmunol.1001188

Malhotra, I., Dent, A., Mungai, P., Wamachi, A., Ouma, J. H., Narum, D. L., et al. (2009). Can prenatal malaria exposure produce an immune tolerant phenotype? A prospective birth cohort study in Kenya. PLoS Med. 6:e1000116. doi: 10.1371/journal.pmed.1000116

Millington, O. R., Di Lorenzo, C., Phillips, R. S., Garside, P., and Brewer, J. M. (2006). Suppression of adaptive immunity to heterologous antigens during Plasmodium infection through hemozoin-induced failure of dendritic cell function. J. Biol. 5 , 1-22. doi: 10.1186/jbiol34

Minigo, G., Woodberry, T., Piera, K. A., Salwati, E., Tjitra, E., Kenangalem, E., et al (2009). Parasite-dependent expansion of TNF receptor II-positive regulatory T cells with enhanced suppressive activity in adults with severe malaria. PLoS Pathog. 5:e1000402. doi: 10.1371/journal.ppat.1000402

Moseman, E. A., Liang, X., Dawson, A. J., Panoskaltsis-Mortari, A., Krieg, A. M., Liu, Y. J., et al. (2004). Human plasmacytoid dendritic cells activated by CpG oligodeoxynucleotides induce the generation of $\mathrm{CD} 4+\mathrm{CD} 25+$ regulatory $\mathrm{T}$ cells. J. Immunol. 173, 4433-4442.

Noland, G. S., Briones, N., and Sullivan, D. J. Jr. (2003). The shape and size of hemozoin crystals distinguishes diverse Plasmodium species. Mol. Biochem. Parasitol. 130, 91-99. doi: 10.1016/S0166-6851(03)00163-4

Orengo, J. M., Wong, K. A., Ocana-Morgner, C., and Rodriguez, A. (2008). A Plasmodium yoelii soluble factor inhibits the phenotypic maturation of dendritic cells. Malar. J. 7:254. doi: 10.1186/1475-2875-7-254

Scholzen, A., Minigo, G., and Plebanski, M. (2010). Heroes or villains? $\mathrm{T}$ regulatory cells in malaria infection. Trends Parasitol. 26, 16-25. doi: 10.1016/j.pt.2009.10.004

Scholzen, A., Mittag, D., Rogerson, S. J., Cooke, B. M., and Plebanski, M. (2009). Plasmodium falciparum-mediated induction of human CD25hiFoxp3hi CD4 T cells is independent of direct TCR stimulation and requires IL-2, IL-10 and TGFbeta. PLoS Pathog. 5:e1000543. doi: 10.1371/journal.ppat.1000543

Shio, M. T., Kassa, F. A., Bellemare, M. J., and Olivier, M. (2010). Innate inflammatory response to the malarial pigment hemozoin. Microbes Infect. 12, 889-899. doi: 10.1016/j.micinf.2010.07.001

Soulard, V., Amadoudji Zin, M., Fitting, C., Ibitokou, S., Oesterholt, M., Luty, A. J., et al. (2011). Placental malaria-associated suppression of parasite-specific immune response in neonates has no major impact on systemic CD4 $\mathrm{T}$ cell homeostasis. Infect. Immun. 79, 2801-2809. doi: 10.1128/IAI.00 203-11

Stevenson, M. M., Ing, R., Berretta, F., and Miu, J. (2011). Regulating the adaptive immune response to blood-stage malaria: role of dendritic cells and CD4(+)Foxp3(+) regulatory $\mathrm{T}$ cells. Int. J. Biol. Sci. 7, 1311-1322. doi: $10.7150 /$ ijbs.7.1311

Walther, B., Miles, D. J., Waight, P., Palmero, M. S., Ojuola, O., Touray, E. S., et al. (2012). Placental malaria is associated with attenuated CD4 T-cell responses to tuberculin PPD 12 months after BCG vaccination. BMC Infect. Dis. 12:6. doi: 10.1186/1471-2334-12-6

Walther, M., Jeffries, D., Finney, O. C., Njie, M., Ebonyi, A., Deininger, S., et al. (2009). Distinct roles for FOXP3 + and FOXP3-CD4+ T cells in regulating cellular immunity to uncomplicated and severe Plasmodium falciparum malaria. PLoS Pathog. 5:e1000364. doi: 10.1371/journal.ppat.1000364

Whittle, H. C., Brown, J., Marsh, K., Greenwood, B. M., Seidelin, P., Tighe, H., et al. (1984). T-cell control of Epstein-Barr virus-infected B cells is lost during P. falciparum malaria. Nature 312, 449-450. doi: 10.1038/312449a0

Williamson, W. A., and Greenwood, B. M. (1978). Impairment of the immune response to vaccination after acute malaria. Lancet 1, 1328-1329. doi: 10.1016/S0140-6736(78)92403-0

Wykes, M. N., and Good, M. F. (2008). What really happens to dendritic cells during malaria? Nat. Rev. Microbiol. 6, 864-870. doi: 10.1038/nrmi cro1988

Conflict of Interest Statement: The authors declare that the research was conducted in the absence of any commercial or financial relationships that could be construed as a potential conflict of interest.

Received: 20 February 2014; accepted: 15 April 2014; published online: 01 May 2014. Citation: Scholzen A, Cooke BM and Plebanski M (2014) Plasmodium falciparum induces Foxp3hi CD4 T cells independent of surface PfEMP1 expression via small soluble parasite components. Front. Microbiol. 5:200. doi: 10.3389/fmicb.2014.00200 This article was submitted to Microbial Immunology, a section of the journal Frontiers in Microbiology.

Copyright (c) 2014 Scholzen, Cooke and Plebanski. This is an open-access article distributed under the terms of the Creative Commons Attribution License (CC BY). The use, distribution or reproduction in other forums is permitted, provided the original author(s) or licensor are credited and that the original publication in this journal is cited, in accordance with accepted academic practice. No use, distribution or reproduction is permitted which does not comply with these terms. 